\title{
Eliminación de Fluoxetina presente en aguas contaminadas usando procesos fotoquímicos de oxidación avanzada y luz solar
}

\author{
$\triangle$ Lis MANRIQUe LOSADA ${ }^{1}$ \\ CAROLINA QuimbaYA ÑANEZ1 \\ Ricardo ANTONio ToRres PALMA ${ }^{2}$
}

\section{Resumen}

El antidepresivo fluoxetina es un contaminante detectado en aguas naturales. En este trabajo se evaluó la degradación de la fluoxetina, en agua destilada (AD) y agua residual municipal (ARM), por medio de tres procesos fotoquímicos de oxidación avanzada (Foto-Fenton-FFS, Foto-Electro-Fenton-FEFS y Fotocatálisis con $\mathrm{TiO}_{2}$-FCS) mediados por luz solar. Para el proceso FEFS, se equipó el reactor con un ánodo de $\mathrm{DSA}\left(\mathrm{Ti} / \mathrm{IrO}_{2}\right.$ dopado con $\mathrm{RuO}_{2}$ ) y un cátodo de grafito de difusión de aire para la producción continua de peróxido. Se hizo seguimiento de la degradación, la mineralización de la fluoxetina y la toxicidad. El proceso FFS presentó una alta eficiencia en la mineralización en comparación a los otros dos procesos FEFS y FCS en AD. El pH definió el alcance de la mineralización de los procesos FFS y FEFS. De hecho, a pH ácido se logró una mayor mineralización, porque se evitó la formación de hidróxidos de hierro (III) típicos a valores de pH mayores. El proceso FCS presenta alta eficiencia de degradación en ARM, alcanzando un 92.6\%, mientras que el proceso FFS disminuyo hasta un $38.5 \%$ de degradación a pH natural. En todos los procesos, la toxicidad ante levadura de cerveza disminuyó a medida que se degradó la Fluoxetina.

Palabras claves: Fluoxetina, Foto-Fenton solar, Foto-Electro-Fenton, Fotocatálisis con $\mathrm{TiO}_{2}$, Luz solar.

1 Universidad de la Amazonia, Florencia Caquetá Colombia.

2 Universidad de Antioquia, Medellín Colombia.

Autor de correspondencia: Manrique-Losada, L. (Lis): Calle 33 - carrera 7, casa 3, Conjunto Calle Real, Barrio la Paz, Florencia-Caquetá, Colombia. Teléfono: 3165394715. Correo electrónico: lismanrique@gmail.com
Historia del artículo:

Artículo recibido: 14-V-2017 / Aprobado: 30-V-2019

Disponible online: 3 de julio de 2019

Discusión abierta hasta marzo de 2021 


\title{
Elimination of fluoxetine present in contaminated water using photochemical processes of advanced oxidation and sunlight
}

\begin{abstract}
The degradation of fluoxetine was evaluated by means of three advanced oxidation photochemical processes: photo-Fenton (SPF), photo-electro-Fenton (SPEF) and photocatalysis with $\mathrm{TiO}_{2}$ (SPC) in distilled water (DW) and municipal wastewater (MWW), mediated by solar radiation. To the SPEF, the reactor was equipped with an DSA ( $\mathrm{Ti}^{\prime} / \mathrm{IrO}_{2}$ doped with $\mathrm{RuO}_{2}$ ) anode and an air diffusion graphite cathode for continuous peroxide production. The mineralization of fluoxetine and toxicity were monitored. The SPF process showed the highest speed of mineralization compared to the other two SPEF and SPC processes in DW. The pH defined the extent of the mineralization of the SPF and SPEF processes, evidencing that acidic $\mathrm{pH}$ achieves greater mineralization by avoiding the formation of complexes and hydroxides typical of a higher pH. The FCS process presents high degradation efficiency in ARM, reaching 92.6\%, while the FFS process decreased up to $38.5 \%$ degradation at natural $\mathrm{pH}$. In all processes, the toxicity to brewer's yeast decreased as Fluoxetine was degraded.
\end{abstract}

Keywords: Fluoxetine, Solar photo-Fenton, Solar photo-electro-Fenton, $\mathrm{TiO}_{2}$ Photocatalysis, Sunlight.

\section{Eliminação de fluoxetina presente em águas contaminadas utilizando fotoquímicas processos de oxidação avançada e luz solar}

\section{Resumo}

A degradação de fluoxetina foi avaliada por meio por três processos avançados de oxidação fotoquímica: foto Fenton (FFS), foto electro Fenton (FEFS) e fotocatálise $\mathrm{TiO}_{2}$ (FCS) em água destilada (DA) e águas residuais municipais (ARM), mediada pela radiação solar. As experiências foram realizadas com soluções de 100 ml num vaso de reacção e no caso de FEFS, o reactor com um ânodo de DSA ( $\mathrm{Ti} / \mathrm{IrO}_{2}$ dopado com $\mathrm{RuO}_{2}$ ) e a difusão do ar do cátodo de grafite para a produção contínua de peróxido foi montado. Degradação de monitorização, a mineralização de fluoxetina e toxicidade era. 0 processo de FFS apresentaram a maior taxa de mineralização, em comparação com os outros dois processos FEFS e FCS no AD. 0 pH definiu a extensão da mineralização dos processos FFS e FEFS, mostrando que o pH ácido é conseguido porque maior mineral a formação do complexo e hidróxidos típicos pH mais elevado é evitado. 0 processo FCS apresenta alta eficiência de degradação em ARM, atingindo 92,6\%, enquanto o processo FFS diminuiu até 38,5\% de degradação em pH natural. Em todos os processos, a toxicidade para a levedura de cerveja diminuiu com a degradação da fluoxetina.

Palavras-chave: Fluoxetina, Foto-Fenton solares, Foto electro Fenton, Fotocatálise $\mathrm{TiO}_{2}$, Radiação solar. 


\section{Introducción}

La fluoxetina (FLX), también conocida por los nombres comerciales Prozac ${ }^{\circledR}$, Sarafem o Fontex, es un antidepresivo fluorado (Figura 1) perteneciente a la clase de inhibidores selectivos de la recaptación de serotonina. La FLX es utilizada para el tratamiento de depresión, ansiedad generalizada, desórdenes obsesivo-compulsivos, bulimia y desorden disfórico premenstrual (Castro et al., 2005; Méndez-Arriaga et al., 2011), (Salazar et al., 2016).

Debido a la gran producción y prescripción de FLX en el mundo entero, su excreción en forma de orina o heces y la ineficiencia de las plantas convecionales de tratamiento de aguas, la presencia del fármaco en suelos, aguas residuales, aguas superficiales e incluso en agua potable ha sido reportada (Su et al., 2013) (Salazar et al., 2016). Es así como la FLX se ha detectado en aguas superficiales de Estados Unidos y Canadá en concentraciones de 0.012 y $0.013-0.046 \mu \mathrm{g} \mathrm{L}^{-1}$, respectivamente; en los efluentes de plantas de tratamiento de aguas a concentraciones de 0.038 - $0.099 \mu \mathrm{g} \mathrm{L}^{-1}$ en Canadá y de $0.0017 \mu \mathrm{g} \mathrm{L}{ }^{-1}$ en Corea del Sur; de 0.0006 - $0.0187 \mu \mathrm{g}$ $\mathrm{L}^{-1}$ en aguas residuales en Noruega; mientras que de $0.021 \mu \mathrm{g} \mathrm{L}^{-1}$ en aguas residuales de Estados Unidos. Adicionalmente, se ha detectado en biosólidos y sedimentos (EE.UU.) (37.4 y $1.84 \mu \mathrm{g} \mathrm{kg}^{-1}$, respectivamente). La FLX también se ha encontrado en el agua potable a concentraciones $<0.014 \mu \mathrm{g} \mathrm{L}^{-1}$ en Estados Unidos (Honda et al., 2016). Se ha reportado la bioacumulación de la FLX en los tejidos (de 0.14 a $1.02 \mu \mathrm{g} \mathrm{kg}^{-1}$ ), en el hígado y el cerebro de peces expuestos a efluentes de agua conteniendo el fármaco (Honda et al., 2016; Zhao et al., 2017).

De otra parte, se ha determinado que la fluoxetina ha mostrado ser tóxica para algas e invertebrados, ya que interactúa con los procesos de crecimiento y reproducción. Además FLX puede inducir el desove en algunos crustáceos y especies bivalvos (MéndezArriaga et al., 2011),(Salazar et al., 2016). Se ha evidenciado que la capacidad de las especies de peces híbridos de róbalo rayado (Morone saxatilis×Morone chrysops) para capturar a sus presas disminuyó después de 6 días de exposición a FLX a concentraciones de 23.2 a $100.9 \mu$ g.L.-1 (Honda et al., 2016).

La FLX reacciona rápidamente con hipoclorito en agua potable y en aguas residuales, formando N-clorofluoxetina (una cloramina), lo que aumenta la hidrofobicidad y la tendencia a adsorberse sobre sedimentos, suelos, y membranas biológicas; estas características contribuyeron a la reciente inclusión de la FLX en la lista de 10 productos farmacéuticos potencialmente peligrosos para el medio ambiente (Honda et al., 2016).

Al ser un fármaco altamente peligroso, la investigación debe dirigirse a encontrar métodos para su eliminación. En este contexto, se ha reportado que la FLX no se puede eliminar por métodos fisicoquímicos y biológicos comúnmente utilizados en plantas de tratamiento de aguas residuales (Salazar et al., 2016).

Por tal razón se han buscado alternativas de oxidación eficientes para la degradación de este antidepresivo, entre los que se pueden citar procesos de oxidación avanzada (POAs) como: Fenton (Honda et al., 2016; Li et al., 2011), foto-Fenton (Perini et al., 2016), ozonólisis (Zhao et al., 2017), degradación por sonoquímica acoplada a tratamiento biológico (Honda et al., 2016) y procesos de oxidación electroquímicos como: oxidación anódica, electro-Fenton y foto-electro-Fenton con luz ultravioleta (Salazar et al., 2016); los cuales han reportado porcentajes de degradación de hasta al 90\%. Dentro de ellos, y en la búsqueda de procesos con bajos requerimientos energéticos, destacan los que emplean la luz solar como fuente gratis de energía lumínica. Se ha demostrado que los procesos asistidos por luz solar promueven la degradación, oxidación y mineralización de compuestos recalcitrantes aun estando éstos en matrices complejas como aguas residuales, además la luz solar como fuente de luz contribuye al desarrollo de tecnologías en el marco de la sostenibilidad (eficientes y económicas).

Los procesos con mayor eficiencia que aprovechan la luz solar para la degradación de contaminantes son fotocatálisis heterogénea, foto-Fenton y 
foto-electro-Fenton. La fotocatálisis heterogénea es comúnmente aplicada para el tratamiento de aguas usando el catalizador Degussa P25 $\left(\mathrm{TiO}_{2}\right)$. El $\mathrm{TiO}_{2}$ es un semiconductor de reconocida eficiencia, que es capaz de foto-activarse a longitudes de onda por debajo de $380 \mathrm{~nm}$, la que se logra con la pequeña porción de luz UV de la luz solar. El proceso fotoFenton solar (FFS) es un proceso catalítico homogéneo donde el mayor contacto del sustrato con el catalizador, suele incrementar la cinética de reacción. Finalmente, en el proceso foto-electro-Fenton solar (FEFS), gracias a una diferencia de potencial en una celda electroquímica, se promueve la formación de peróxido en el cátodo, el cual en presenccia de hierro genera las especies oidantes, al tiempo que la oxidación anódica contribuye también a la degradación de los contaminantes.

Basado en lo anterior, el objetivo de esta investigación es evaluar y comparar la degradación de la FLX por medio de tres procesos fotoquímicos de oxidación avanzada: foto-Fenton (FFS), fotocatálisis heterogénea con $\mathrm{TiO}_{2}$ (FCS) y foto-electro-Fenton (FEFS), todos promovidos por luz solar. Los procesos fotoquímicos seleccionados se caracterizan por ser diversos en su montaje, operación y disposición de los reactantes involucrados. En el proceso FCS, se evaluará el efecto de la presencia de peróxido de hidrógeno y la dosis del catalizador sobre la degradación y mineralización de la FLX, adicionalmente se definirá la posible ruta de degradación. En la aplicación de FFS se definirá la influencia de la concentración de $\mathrm{Fe}^{+2}$ y de $\mathrm{H}_{2} \mathrm{O}_{2}$ y las diferencias en degradación ante diferente $\mathrm{pH}$; mientras que durante la aplicación de FEFS se determinará el aporte de la oxidación anódica, electro-Fenton y foto-electroFenton a la degradación de FLX.

\section{Métodos y materiales}

\subsection{Reactivos}

La FLX fue suministrada por Allianz Group International Ltda. El ácido sulfúrico, el acetonitrilo (ACN), el bisulfito de sodio, el sulfato anhídrido de so- dio fue suministrado por Merck®, el $\mathrm{TiO}_{2}$ usado fue Degussa P25 de Evonik® y el peróxido de hidrogeno y yoduro de potasio (KI) comprado a Panreac®.

Figura 1. Estructura química de la Fluoxetina

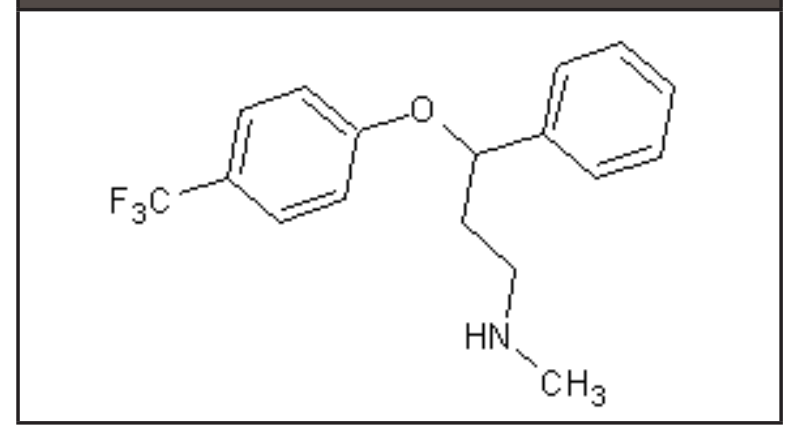

\subsection{Zona de muestreo}

La muestra de agua residual municipal (ARM) fue recolectada en las siguientes coordenadas “N 136'51,805" $075^{\circ} 36^{\prime} 29,036^{\prime}$, una altura de $261 \mathrm{msnm}$, en un vertimiento directo de aguas residuales provenientes de los barrios Ventilador, Acacias, Simón Bolívar, Los Alpes, Buenos Aires, Raicero, Comuneros, La Bocana, El Guamal y Juan XXIII bajo, de la ciudad Florencia (Caquetá, Colombia) (SERVAF \& Alcaldia Florencia, 2013).

\subsection{Sistemas de reacción}

La degradación de FLX (40 mg L $\left.{ }^{-1}\right)$ por FFS de $100 \mathrm{~mL}$ de agua simulada preparada con agua destilada y FLX, se realizó a diferentes concentraciones de $\mathrm{Fe}^{2+}(1.8,9$ y $90 \mu \mathrm{M})$ con $1000 \mu \mathrm{M}$ de $\mathrm{H}_{2} \mathrm{O}_{2}$ en agitación constante durante $2 \mathrm{~h}$. Estos experimentos se realizaron a pH ácido (3.08) y pH natural (6.0). El $\mathrm{H}_{2} \mathrm{O}_{2}$ se eliminó utilizando bisulfito de sodio 0.1 $\mathrm{M}$ en todos los procesos donde se utilizó. Antes de realizar la degradación de la FLX en ARM, la muestra de agua se filtró con un papel filtro cualitativo, posteriormente se adiciono la FLX a una concentración de $40 \mathrm{mg} \mathrm{L}^{-1}$ en este experimento se utilizó $\mathrm{Fe}^{2+}(18$ $\mu \mathrm{M}$ ) con $1000 \mu \mathrm{M}$ de $\mathrm{H}_{2} \mathrm{O}_{2}$ a pH natural.

En el proceso fotocatalítico se evaluó el efecto de la dosis de catalizador en suspensión $(50,100$, 300 y $500 \mathrm{mg} \mathrm{L}^{-1} \mathrm{de} \mathrm{TiO}_{2}$ ) y la concentración de $\mathrm{H}_{2} \mathrm{O}_{2}$ (1000 $\mu \mathrm{M}$ y $4409 \mu \mathrm{M} \mathrm{H}_{2} \mathrm{O}_{2}$ con $50 \mathrm{mg} \mathrm{L}^{-1} \mathrm{TiO}_{2}$ ). En la 
degradación fotocatalítica se utilizó 100 mL de FLX ( $40 \mathrm{mg} \mathrm{L}^{-1}$ ) en agitación constante durante $2 \mathrm{~h}$. Para determinar la contribución de los huecos fotogenerados y los radicales hidroxilos en la degradación de la FLX se utilizó KI y ACN, respectivamente, con una concentración de $\mathrm{TiO}_{2}$ de $500 \mathrm{mg} \mathrm{L}^{-1}$. El KI se adicionó al sistema de reacción para que quedara a una concentración diez veces mayor a la del contaminante. El ACN se utilizó como disolvente de la FLX. Se utilizó $500 \mathrm{mg} \mathrm{L}^{-1}$ de $\mathrm{TiO}_{2}$ para el tratamiento $\mathrm{FCS}$ de ARM a pH natural.

Los experimentos de oxidación anódica se llevaron a cabo en una celda electrolítica que contenía 150 mL de FLX, en agitación constante. Se utilizó un ánodo de $\mathrm{DSA}\left(\mathrm{Ti} / \mathrm{IrO}_{2}\right.$ dopado con $\mathrm{RuO}_{2}$ ) y un cátodo de difusión de aire. Los electrodos se ubicaron en el centro del reactor a una distancia de $3.0 \mathrm{~cm}$, y fueron operados a corriente constante $(3.25 \mathrm{~mA}$ $\mathrm{cm}^{-2}$ ). Como electrolito soporte se usó $\mathrm{Na}_{2} \mathrm{SO}_{4}$ a 0.05 mol L-1. Los experimentos de electro-Fenton (EF) y foto-electro-Fenton (FEFS) se realizaron a $\mathrm{pH}$ acido (3.08) y pH natural (6.0) con una concentración 18 $\mu \mathrm{M}$ de $\mathrm{Fe}^{2}$. Para el tratamiento FEFS de ARM a $\mathrm{pH}$ natural se utilizó $18 \mu \mathrm{M}$ de $\mathrm{Fe}^{2}$.

La luz solar se midió con un radiómetro Solar Light PMA2100 Data Logging con sensores para UV y luz visible acoplados. La relación entre tiempo de exposición solar y energía acumulada se calculó con la expresión (Ecuación 1):

$$
\mathrm{Ea}=\sum_{\mathrm{i}=0}^{\mathrm{n}} \frac{\mathrm{I} \Delta \mathrm{t}_{\mathrm{i}}}{\mathrm{V}} a
$$

Donde I es el flujo de fotones incidente $\left(\mathrm{W}^{2} \cdot \mathrm{s}^{-1}\right)$, $\Delta \mathrm{t}_{\mathrm{i}}$ es el tiempo entre muestras (s), $\mathrm{V}$ es el volumen de la solución tratada (L) y (a) área iluminada del reactor.

\subsection{Seguimiento de degradación y mineralización}

El seguimiento de la degradación de la FLX en agua destilada se realizó por espectrofotometría UV/VIS. El seguimiento de degradación del producto farmacéutico en presencia de $\mathrm{KI}, \mathrm{ACN}$ y en ARM se realizó por HPLC usando una columna C-18 (5 $\mu \mathrm{m}$ dp $250 \times 4,6 \mathrm{~mm}$ ) y un detector UV a $227 \mathrm{~nm}$. La fase móvil fue un tampón de ácido fórmico $(10 \mathrm{mM} \mathrm{pH}$ $3.0) /$ acetonitrilo $40 / 60(\% \mathrm{v} / \mathrm{v})$, en modo isocrático $\left(1 \mathrm{~mL} \mathrm{~min}^{-1}\right)$. La mineralización de la muestra se siguió mediante análisis de carbono orgánico total (COT) determinado por el método de combustión catalítica utilizando un automuestreador Shimadzu TOC-L + ASI-L. Se utilizó una solución de ftalato de potasio como estándar de calibración y el volumen de inyección fue de $50 \mu \mathrm{L}$. La toxicidad de la solución tratada en agua destilada se realizó frente a Saccharomyces cerevisiae (levadura de cerveza) mediante un recuento microscópico en un una Cámara de $\mathrm{Ne}$ ubauer, por conteo de células muertas.

\section{Resultados y discusión}

\subsection{Degradación de la FLX por foto- catálisis heterogénea con $\mathrm{TiO}_{2}$ (FCS)}

Con el fin de definir diferencias entre la eficiencia de degradación y mineralización de la FLX por FCS, se llevó a cabo el proceso bajo cuatro dosis de catalizador. Las dosis más altas de catalizador mejoraron la velocidad de degradación si se comparan con la velocidad a dosis menores como $50 \mathrm{y}$ 100 ppm de $\mathrm{TiO}_{2}$ (Figura 2). Para las condiciones de reacción en el laboratorio, trabajar con 300 o 500 ppm de $\mathrm{TiO}_{2}$, implica la misma respuesta en cinética de degradación. En cuanto a la mineralización se observó que con el incremento de la concentración de $\mathrm{TiO}_{2}$ el porcentaje de mineralización aumenta, esto puede ocurrir debido a que se genera mayor cantidad de radicales $\mathrm{HO}$ y hay mayor disposición de superficies activas $\left(\mathrm{h}^{+}\right)$para oxidar el agua y mineralizar la materia orgánica. Se alcanzó una mineralización de hasta $68.9 \%$ con 500 ppm de $\mathrm{TiO}_{2}$ (Tabla 1). Trabajar por encima de concentraciones de $500 \mathrm{mg}$ $\mathrm{L}^{-1}$ podría generar apantallamiento y evitaría el paso del haz de luz hasta la superficie del catalizador (Giraldo et al., 2010). Es interesante observar el comportamiento del TOC en contraste con la degradación cuando la dosis de $\mathrm{TiO}_{2}$ es $500 \mathrm{mg} \mathrm{L}^{-1}$. Después 
de alcanzar una energía acumulada de alrededor de $18 \mathrm{~kJ} \mathrm{~L}^{-1}$ el TOC disminuye drásticamente, evidenciando que solo después de la degradación del $68 \%$ de FLX, los subproductos de degradación de FLX sufren oxidación hasta $\mathrm{CO}_{2}$. Adiconalmente, este comportamiento donde la degradación y mineralización transcurren casi en forma paralela, implica un uso eficiente de la energía solar acumulada.
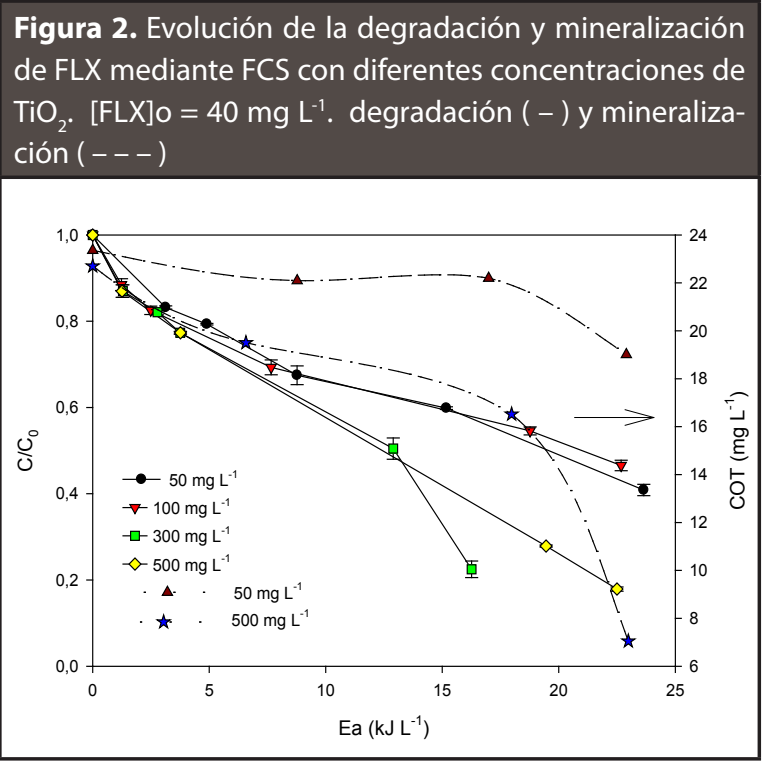

Después de conocer la dosis de catalizador que presenta el mayor alcance de degradación y mineralización sin llegar al apantallamiento, es necesario definir una posible ruta de degradación de la FLX cuando se somete a este proceso. Teniendo en cuenta que principalmente son dos rutas posibles: oxidación foto-Kolbe y/o oxidación radicalaria, se desarrollaron experimentos de control cuyos resultados se muestran en la Figura 3. La adsorción de la FLX sobre el catalizador es nula durante dos horas, ya que la FLX presenta un pKa $=8.7$ (Zhao et al., 2017) y si se considera que el $\mathrm{pH}$ de trabajo siempre fue el natural (cercano a 6), la superficie del catalizador permaneció en forma catiónica al igual que la especiación de la FLX, por tal razón no se evidencia adsorción del contaminante en la superficie del catalizador. Cuando se adiciona KI que es un scavenger de $\mathrm{h}^{+}$en la superficie, la degradación de FLX alcanzo el 49\%, ésta respuesta implica que hay un aporte de los huecos $\left(\mathrm{h}^{+}\right.$, foto Kolve) a la degradación de FLX (Giraldo-Aguirre et al., 2015). El anterior comportamiento unido a la respuesta de la degradación cuando hay acetonitrilo que es un scavenger de radicales, donde la degradación alcanzó un 46.7\%, confirma que la degradación de la FLX se degrada vía fotoKolbe y radicalaria al mismo tiempo.

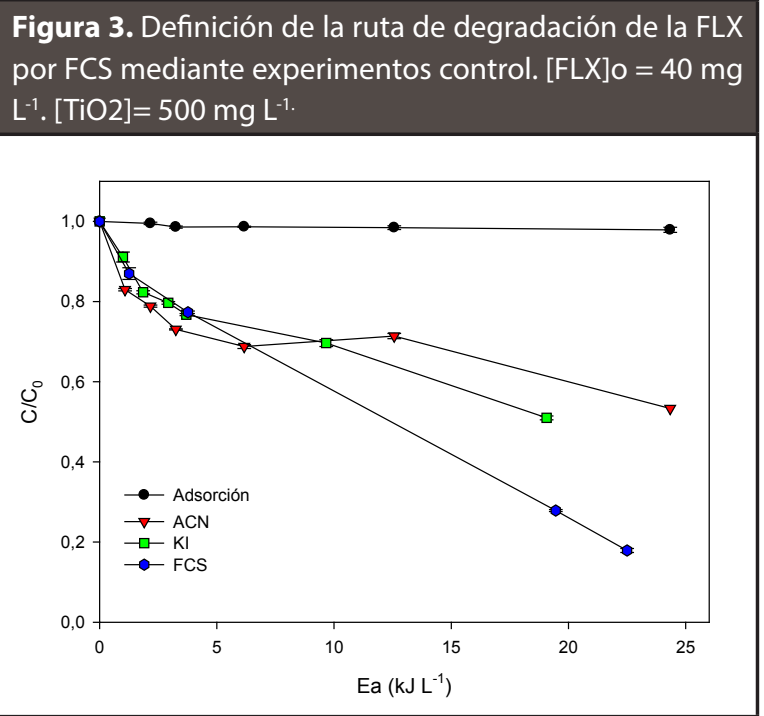

La literatura reporta ampliamente la influencia positiva de un agente oxidante como el $\mathrm{H}_{2} \mathrm{O}_{2}$ en un sistema de reacción por FCS. El papel del $\mathrm{H}_{2} \mathrm{O}_{2}$ consiste en evitar la recombinación de pares electrón hueco y en su capacidad de reacción con electrones fotogenerados para formar otras especies radicalarias y posteriormente radicales $\mathrm{HO} \bullet$. La Figura 4 presenta la respuesta de la degradación de FLX a una concentración de $50 \mathrm{mg} \mathrm{L}^{-1}$ cuando se agrega $\mathrm{H}_{2} \mathrm{O}_{2}$ como promotor de mayor concentración de radical hidroxilo. La presencia de peróxido de hidrogeno modificó notoriamente la degradación de la FLX, aumentándola desde 59\% a 75\%, cuando se adicionó una concentración de $1000 \mu \mathrm{M}$ de $\mathrm{H}_{2} \mathrm{O}_{2}$ a $50 \mathrm{mg} \mathrm{L}^{-1}$ de $\mathrm{TiO}_{2}$. En contraste, el porcentaje de remoción de la FLX disminuyó de 59\% a 51\%, cuando se adicionó $4409 \mu \mathrm{M}$ de $\mathrm{H}_{2} \mathrm{O}_{2}$. La degradación de FLX con [ $\left.\mathrm{TiO}_{2}\right]$ $=50 \mathrm{mg} \mathrm{L}^{-1}$ presento una constante de velocidad de degradación (k) de $3.52 \times 10^{-2} \mathrm{~s}^{-1}$, mientras que con una dosis de $\mathrm{H}_{2} \mathrm{O}_{2}$ mayor $(4409 \mu \mathrm{M})$ no se vio afecta- 
da e incluso disminuyo $\left(\mathrm{k}=2.69 \times 10^{-2}\right)$ (Ver Tabla 1). Éste comportamiento se puede atribuir a que el peróxido cuando está en exceso se comporta como un scavenger de radicales (Ecuaciones 2 y 3 ) (Agudelo, 2007).

Figura 4. Evolución de la degradación y mineralización FLX mediante FCS y diferentes concentraciones de $\mathrm{H}_{2} \mathrm{O}_{2}$. $[F L X]=40 \mathrm{mg} \mathrm{L}-1 .\left[\mathrm{TiO}_{2}\right]=50 \mathrm{mg} \mathrm{L-1}$. Degradación ( - ). Mineralización (-- - )

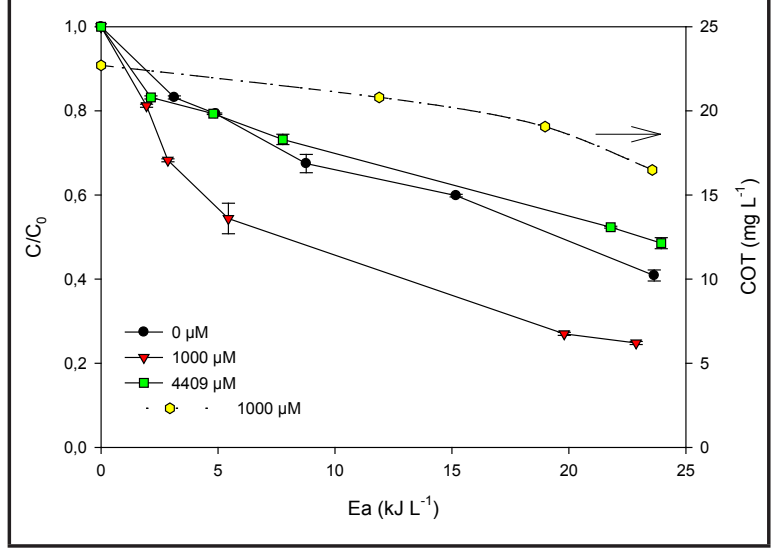

$$
\begin{gathered}
\mathrm{H}_{2} \mathrm{O}_{2}+\mathrm{OH} \bullet \rightarrow \mathrm{OH}_{2}^{\bullet}+\mathrm{H}_{2} \mathrm{O} \\
\mathrm{OH}_{2}^{\bullet}+\mathrm{OH} \bullet \rightarrow \mathrm{H}_{2} \mathrm{O}+\mathrm{O}_{2}
\end{gathered}
$$

Cuando la dosis es menor $(1000 \mu \mathrm{M})$ hay un exceso de peróxido que favorece la degradación debido a que el papel del peróxido en esta situación es promover la generación de más radicales hidroxilos y evitar la recombinación de los pares $\mathrm{e}^{-}-\mathrm{h}^{+}$ (Giraldo-Aguirre et al., 2015; R. A. Luna et al., 2013; Villegas-Guzman et al., 2015).

\subsection{Degradación de la FLX por foto- Fenton (FFS)}

Para iniciar el análisis de los resultados alrededor de degradación de FLX mediante FFS, se requería evaluar y luego comparar esta respuesta con los respectivos procesos de control tales como proceso Fenton a pH natural y ácido, proceso solo con $\mathrm{H}_{2} \mathrm{O}_{2} \mathrm{y}$ proceso con solo $\mathrm{Fe}^{+2}$.
Figura 5. Evolución de la degradación de la FLX mediante Fenton y FFS a pH ácido y $\mathrm{pH}$ natural. $[\mathrm{FLX}] \mathrm{o}=40 \mathrm{mg}$ $\mathrm{L}^{-1},\left[\mathrm{H}_{2} \mathrm{O}_{2}\right] \mathrm{o}=1000 \mu \mathrm{M}$. A) Controles con $\left[\mathrm{Fe}^{+2}\right] \mathrm{o}=9 \mu \mathrm{M}$. B) Fenton a diferentes concentraciones de $\mathrm{Fe}^{+2}$. C) FFS a diferentes concentraciones de $\mathrm{Fe}^{+2}$.
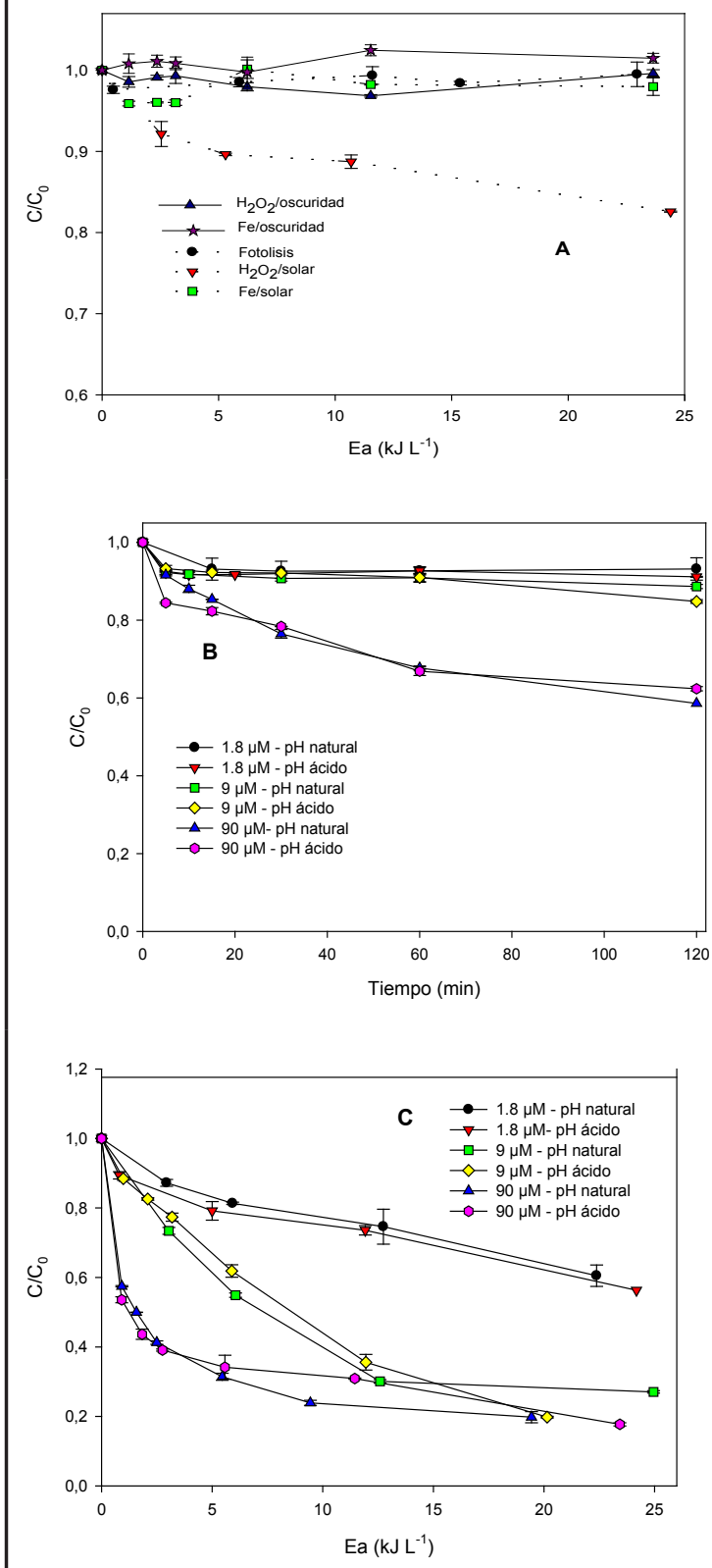

En este contexto, se observa en la Figura 5A, que el $\mathrm{H}_{2} \mathrm{O}_{2}$ en la oscuridad no tiene capacidad oxidante sobre la FLX y que el Fe ${ }^{+2}$ no remueve el contaminante, por tanto, no ocurrió coagulación de materia orgánica con la sal de $\mathrm{Fe}^{+2}$. El proceso Fenton presentó 
una mayor eficiencia en la remoción de la FLX (\% remoción 41.8) con $90 \mu \mathrm{M}$ de $\mathrm{Fe}^{2+}$ y 1000 de $\mu \mathrm{M}$ de $\mathrm{H}_{2} \mathrm{O}_{2}$ a pH natural después de 120 minutos de reacción. A pH ácido, bajo estas mismas condiciones, alcanzó un $37.6 \%$ de degradación (Figura 5B). Con $9 \mu \mathrm{M}$ de $\mathrm{Fe}^{2+}$, se observó una disminución de la remoción de FLX, alcanzando un $15.2 \%$ a pH ácido y un $11.4 \%$ a pH natural. Finalmente, a $1.8 \mu \mathrm{M}$ de $\mathrm{Fe}^{2+}$ la degradación de la FLX fue menor en comparación a las otras dosis de $\mathrm{Fe}^{2+}$ utilizadas, con un $8.8 \%$ y $6.8 \%$ de degradación a $\mathrm{pH}$ ácido y natural, respectivamente (Figura 5B).

Las concentraciones de $\mathrm{Fe}^{+2}$ muy bajas $\left(\left[\mathrm{Fe}^{+2}\right]\right.$ $<90 \mu \mathrm{M}$ ) no ofrecieron la concentración de catalizador suficiente para llevar a cabo la reacción Fenton. Se generó, por tanto, una baja concentración de radicales hidroxilos y esto sumado a la dificultad de la regeneración del catión de $\mathrm{Fe}^{+2}$ a partir del $\mathrm{Fe}^{+3}$ ocasionó una cinética muy lenta y una baja eficiencia de degradación. Por otro lado, se evidenció una cinética de degradación similar a pH natural y pH ácido lo que implicaría que la interacción radicalaria con la FLX es lo suficientemente rápida en cualquier $\mathrm{pH}$, dado que durante la reacción estuvo presente la forma protonada de la FLX. Esa interacción radicalaria a pH natural podría ser atribuida a que se generaron radicales hidroxilos que promovieron la degradación inicial y después los productos intermediarios causaron un descenso drástico en el pH (no reportado en este artículo) y por tanto se favoreció la degradación aún más.

En el proceso Fenton la regeneración del catión $\mathrm{Fe}^{2+}$ es lenta y está acompañada por la producción de radicales hidroperóxilo $\left(\mathrm{HO}_{2}\right.$ ) ( $\mathrm{Li}$ et al., 2011). Al combinar la reacción Fenton con luz solar, se mejora la eficiencia del proceso Fenton, ya que la regeneración del $\mathrm{Fe}^{2+}$ es más rápida y se acompaña de la producción adicional de radicales hidroxilos (Feng \& Le-cheng, 2004; Li et al., 2011), por tal razón se observó un incremento en la velocidad de degradación de la FLX (Figura 5B).

La Figura 5C, muestra que los resultados obtenidos de la degradación de la FLX, $90 \mu \mathrm{M}$ de $\mathrm{Fe}^{2+}$ y $1000 \mu \mathrm{M} \mathrm{de} \mathrm{H}_{2} \mathrm{O}_{2}$, fueron los de mayor eficiencia y cinética de degradación (Tabla 1) y demuestran también que no hay diferencias significativas entre los porcentajes de degradación a $\mathrm{pH}$ ácido o $\mathrm{pH}$ natural (82.3\% y $80.2 \%$ respetivamente). A $1.8 \mu \mathrm{M}$ de $\mathrm{Fe}^{2+}$ la degradación de la FLX fue menor en comparación a las otras dosis de $\mathrm{Fe}^{2+}$ utilizadas. No se evidenció una diferencia significativa cuando se realizó a pH ácido y natural, con un 44\% y 40\% de degradación con energías acumuladas de 24.17 y 22.37 kJ $\mathrm{L}^{-1}$, respectivamente. Al comparar la Figura 5B con la Figura 5C, la presencia de la luz solar mejoró la eficiencia de la degradación en aproximadamente un $35 \%$, y por supuesto la velocidad de degradación (Tabla 1). Lo que indica que la luz solar promovió la reducción del $\mathrm{Fe}^{+3}$ (Affam et al., 2014; De la Cruz et al., 2012; Klamerth et al., 2013).

Los resultados obtenidos en la Figura 5A muestran que la FLX no sufrió fotólisis. La combinación de la FLX con $\mathrm{H}_{2} \mathrm{O}_{2}$ de $1000 \mu \mathrm{M}$ y luz solar (Solar $/ \mathrm{H}_{2} \mathrm{O}_{2}$ ) mejoró la degradación (\% remoción $17 \%$ ) en comparación con la fotólisis. De acuerdo a los resultados mostrados en esta figura, la ruta de degradación por el acople FFS fue principalmente por vía radicalaria gracias a la reacción Fenton con luz solar.

Es importante para la evaluación del proceso, determinar el alcance de la mineralización logrado a las diferentes concentraciones de $\mathrm{Fe}^{+2}$ trabajadas en FFS (resultados no presentados en este trabajo). Se encontró que a $9 \mu \mathrm{M}$ de $\mathrm{Fe}^{2+}$ se logró un $44 \%$ de mineralización a $\mathrm{pH}$ ácido y un $25 \%$ a pH natural, con energías acumuladas de 20.14 y $23.95 \mathrm{~kJ} \mathrm{~L}^{-1}$ respetivamente. A pesar de alcanzar degradaciones cercanas a las dos condiciones de $\mathrm{pH}$ (Figura 5C), la mayor mineralización de la FLX se dio a pH ácido. Es claro que, a pH natural el $\mathrm{Fe}^{2+}$ que alcanza a estar en solución por un tiempo corto, logra vía radicalaria, el rompimiento de enlaces que obligan a la aparición de subproductos de degradación, sin embargo, ellos no se mineralizan. Éste comportamiento se pudo atribuir a la formación de los complejos de hidróxidos que se presentan a $\mathrm{pH}$ por encima de 3 (Bandala et al., 2010; Klamerth et al., 2013). A $90 \mu \mathrm{M}$ de Fe ${ }^{2+}$ también se observó la influencia del pH en la mineralización, alcanzando un $82.7 \%$ a pH ácido y un $65.2 \%$ 
a pH natural (Tabla 1). Finalmente, a $1.8 \mu \mathrm{M}$ de $\mathrm{Fe}^{2+}$ se obtuvo un $24 \%$ (pH ácido) y un $10 \%$ (pH natural) de mineralización. Estos comportamientos pueden atribuirse a que, aunque la luz solar promueve la formación de especies de $\mathrm{Fe}^{+2}$ y de radicales $\mathrm{OH}^{*}$ y evita la acumulación de hidróxidos, la concentración extra de radicales es suficiente para degradación mas no para llegar a mineralización.

\subsection{Degradación de la FLX por foto- electro-Fenton solar}

Cuando se sometió la FLX a degradación vía electroquímica, se evaluaron las diferencias en las cinéticas de degradación con cada uno de los procesos que lo componen: electro-Fenton (EF), oxidación anódica (OA) y FEFS.

Se observa en la Figura 6 que por medio de oxidación anódica no se alcanza degradación de la FLX, indicando que los radicales hidroxilos generados estaban quimi-adsorbidos ocupándose principalmente en la formación de un óxido superior que no fue eficiente en la degradación y mineralización de la materia orgánica, éste comportamiento es típico de un ánodo tipo DSA como el usado en éste trabajo que consiste de la combinación de dos óxidos metálicos activos (Flores et al., 2015; Salazar et al., 2016). Si se implementara un ánodo no activo como por ejemplo BDD, que promueve la formación y fisiadsorción de radicales $\mathrm{OH}^{*}$ en su superficie, se podría mejorar la degradación y mineralización de FLX (Salazar et al., 2017). La Figura 6 también demuestra que al aplicar EF al sistema, se mejora la oxidación a un pH ácido, lo que implica que la degradación es vía únicamente Fenton y que probablemente a $\mathrm{pH}$ natural se formaron los complejos orgánicos o hidroxiacuosos de $\mathrm{Fe}^{+3}$.

La respuesta de la degradación y mineralización de FLX cuando fue sometida a FEFS. La degradación a pH ácido, superó en velocidad a la degradación a pH natural. Sin embargo, la velocidad de degradación en ambos sistemas, es cercana en magnitud: $68.8 \%$ a pH ácido y $67.4 \%$ pH natural (Tabla 1). Éste comportamiento es similar al del proceso FFS de la FLX. De igual manera, la mineralización se vio me- jorada cuando el pH es ácido. Estos resultados indicaron que usando FEFS con un ánodo de $\mathrm{IrO}_{2} / \mathrm{RuO}_{2}$ la ruta de degradación no cambia por el acople electroquímico y se desarrolla únicamente por FFS. Este resultado está acorde con lo evidenciado en trabajos similares, tal como el desarrollado para la degradación de oxacilina vía tratamiento electroquímico en condiciones de laboratorio (Serna-galvis et al., 2016).

Figura 6. Evolución de la degradación y mineralización de la FLX durante OA, EF y FEFS a pH natural y ácido. [FLX]o $=40$ $\mathrm{mg} \mathrm{L}^{-1},\left[\mathrm{Fe}^{2+}\right]=18 \mu \mathrm{M}$. Degradación (-). Mineralización (․).

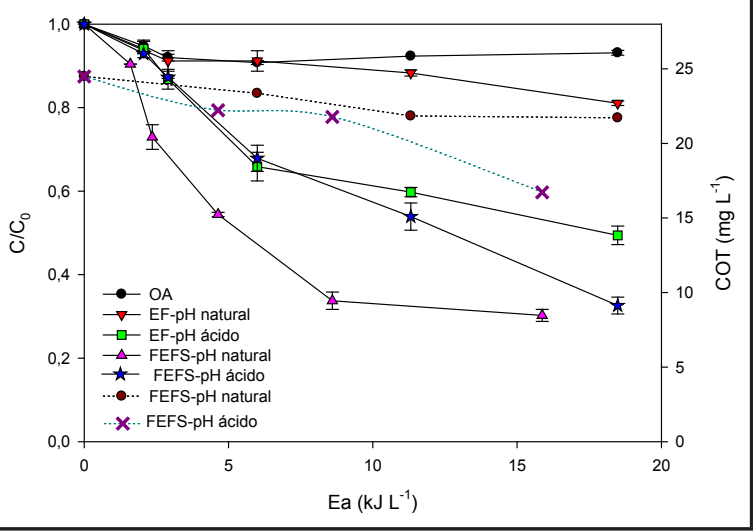

\subsection{Toxicidad de la FLX y sus subpro-} ductos de degradación

Como una forma de aproximar la toxicidad de la FLX y de sus subproductos de degradación, se aplicó un bioensayo donde la levadura de cerveza se puso en contacto con agua tratada mediante con cada de uno de los procesos aplicados. La respuesta al porcentaje de mortalidad en diferentes porcentajes de degradación de la FLX (Figura 8), confirmó que por medio de FFS se alcanzó una caída del 80\% de la mortalidad de la levadura cuando se había degradado solo el 20\% de la FLX. Para los tres procesos restantes hubo un descenso significativo que se pronunció marcadamente cuando se había degradado más del $40 \%$. Se observó que por los tres procesos e incluso por fotocatálisis con $\mathrm{TiO}_{2}$ en presencia de peróxido, se redujo el porcentaje de mortalidad hasta un $20 \%$. A medida que se degradó la FLX, los subproductos de degradación no incrementaron la toxicidad. 
Figura 7. Mortalidad de s. cerevisiae en agua tratada mediante los procesos FFS, FCS con $\left[\mathrm{H}_{2} \mathrm{O}_{2}\right] \mathrm{o}=1000 \mu \mathrm{M}$ y FEFS aplicados para la degradación de la FLX.

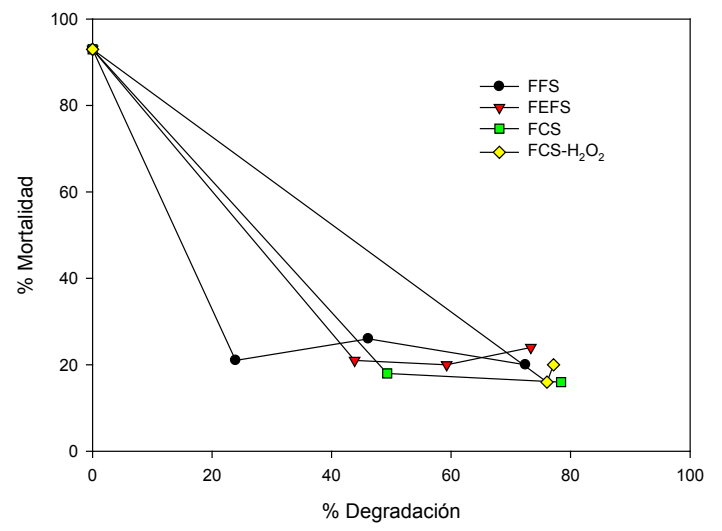

\subsection{Comparación de eficiencias de} degradación de FLX por tres procesos de oxidación avanzada.

Teniendo en cuenta que se utilizó el mismo sistema de reacción para la degradación de la FLX en los tres procesos (concentración inicial de FLX = $40 \mathrm{mg} \mathrm{L}^{-1}$ en sistemas discontinuos de $250 \mathrm{~mL}$, dos horas de reacción y luz solar), es posible hacer una comparación de eficiencias de degradación y mineralización y de sus constantes cinéticas. La Tabla 1 muestra ésta comparación de los tres procesos en agua destilada, en el proceso FFS se observó la velocidad de degradación más alta y el mayor porcentaje de degradación y mineralización. El proceso FCS presentó a mineralización que FFS a pH natural. Dentro de los tres procesos FEFS, presento el menor porcentaje de mineralización, para mejorar este proceso se debe utilizar un ánodo que genere un aporte a la oxidación y mineralización por ejemplo un ánodo BDD (Serna-galvis et al., 2016), no es viable considerar éste proceso para el tratamiento de aguas residuales municipales, ya que se requiere la adición de un electrolito soporte, lo que genera un costo adicional.

Figura 8. Comparación de la degradación de la FLX presente en ARM, alcanzada en los procesos FCS, FFS, FEFS. Condiciones: para FCS: $\left[\mathrm{TiO}_{2}\right] \mathrm{o}=500 \mathrm{mg} \mathrm{L}^{-1}$; para FFS: $\left[\mathrm{Fe}^{+2}\right] \mathrm{o}=18 \mu \mathrm{M}$ y $\left[\mathrm{H}_{2} \mathrm{O}_{2}\right]=1000 \mu \mathrm{M}$ a pH natural y para FEFS: $\left[\mathrm{Fe}^{+2}\right] \mathrm{o}=18$ $\mu \mathrm{M}$ a pH natural.

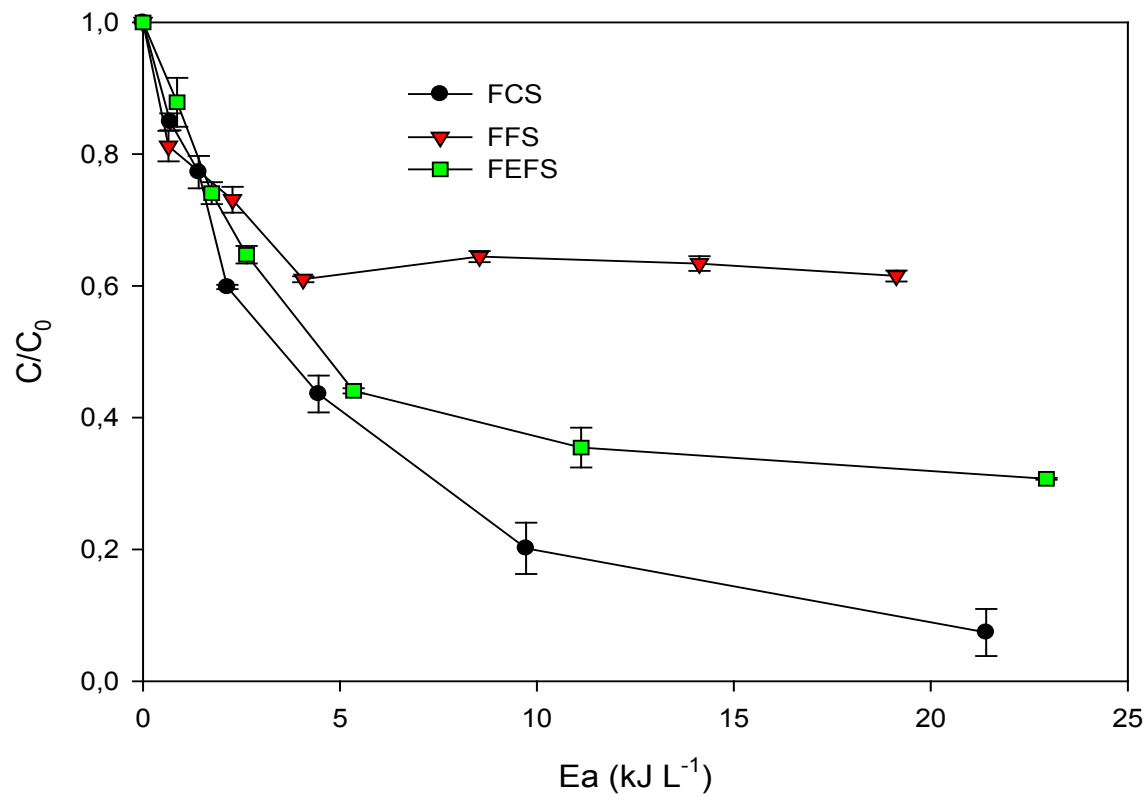


TABLA 1. COMPARACIÓN DE CONSTANTES CINÉTICAS DE DEGRADACIÓN Y MINERALIZACIÓN PARA LOS PROCESOS FFS, FEFS Y FCS.

\begin{tabular}{|c|c|c|c|c|c|}
\hline PAOs & Condiciones & $k\left(s^{-1}\right)$ & \% degradación & $k\left(s^{-1}\right)$ & \% mineralización \\
\hline \multicolumn{6}{|c|}{$A D$} \\
\hline \multirow{2}{*}{ FFS } & $\begin{array}{c}40 \mathrm{mg} \mathrm{L}^{-1} \text { de FLX } \\
{\left[\mathrm{H}_{2} \mathrm{O}_{2}\right]=1000 \mathrm{mM}} \\
{\left[\mathrm{Fe}^{2+}\right]=90 \mu \mathrm{M}} \\
\mathrm{pH}=3.08\end{array}$ & $3.28 \times 10^{-1}$ & $82.3 \%$ & $7.92 \times 10^{-2}$ & $82.7 \%$ \\
\hline & $\begin{array}{c}40 \mathrm{mg} \mathrm{L}^{-1} \text { de FLX } \\
{\left[\mathrm{H}_{2} \mathrm{O}_{2}\right]=1000 \mathrm{mM}} \\
{\left[\mathrm{Fe}^{2+}\right]=90 \mu \mathrm{M}} \\
\mathrm{pH}=\text { natural }\end{array}$ & $3.45 \times 10^{-1}$ & $80.2 \%$ & $7.64 \times 10^{-2}$ & $65.2 \%$ \\
\hline FCS & $\begin{array}{l}40 \mathrm{mg} \mathrm{L}^{-1} \text { de FLX- } \\
{\left[\mathrm{TiO}_{2}\right]=500 \mathrm{mg} \mathrm{L}^{-1}}\end{array}$ & $7.15 \times 10^{-2}$ & $82.1 \%$ & $4.28 \times 10^{-2}$ & $68.9 \%$ \\
\hline \multirow[b]{2}{*}{ FEFS } & $\begin{array}{c}40 \mathrm{mg} \mathrm{L}^{-1} \text { de FLX }\left[\mathrm{Fe}^{2+}\right] \\
=18 \mu \mathrm{M} \\
\mathrm{pH}=3.08\end{array}$ & $1.31 \times 10^{-1}$ & $69.8 \%$ & $2.3 \times 10^{-2}$ & $31.7 \%$ \\
\hline & $\begin{array}{c}40 \mathrm{mg} \mathrm{L}^{-1} \text { de FLX } \\
{\left[\mathrm{Fe}^{2+}\right]=18 \mu \mathrm{M}} \\
\mathrm{pH}=\text { natural }\end{array}$ & $6.09 \times 10^{-2}$ & $67.4 \%$ & $1.01 \times 10^{-2}$ & $11.4 \%$ \\
\hline \multicolumn{6}{|c|}{ ARM } \\
\hline FFS & $\begin{array}{c}40 \mathrm{mg} \mathrm{L}^{-1} \text { de FLX } \\
{\left[\mathrm{Fe}^{2+}\right]=18 \mu \mathrm{M}} \\
\mathrm{pH}=\text { natural }\end{array}$ & $1.09 \times 10^{-1}$ & $38.5 \%$ & NM & NM \\
\hline FCS & $\begin{array}{l}40 \mathrm{mg} \mathrm{L}^{-1} \text { de FLX } \\
{\left[\mathrm{TiO}_{2}\right]=500 \mathrm{mg} \mathrm{L}^{-1}}\end{array}$ & $1.87 \times 10^{-1}$ & $92.6 \%$ & NM & NM \\
\hline FEFS & $\begin{array}{c}40 \mathrm{mg} \mathrm{L}^{-1} \text { de } \mathrm{FLX} \\
{\left[\mathrm{Fe}^{2+}\right]=18 \mu \mathrm{M}} \\
\mathrm{pH}=\text { natural }\end{array}$ & $1.54 \times 10^{-2}$ & $69.3 \%$ & NM & NM \\
\hline \multicolumn{6}{|c|}{$\mathrm{NM}=$ No medido } \\
\hline
\end{tabular}

Los efectos de la matriz en la degradación de la FLX mediante los tres procesos FFS, FEFS y FCS se pueden observar en la Figura 8. En el proceso FFS la presencia de otros compuestos orgánicos e inorgánicos, aniones presentes en el agua, como nitratos, sulfatos, cloruros y fosfatos, pueden reaccionar con hierro $\mathrm{y}_{2} \mathrm{O}_{2}$ u otras especies reactivas de oxí- geno, lo que limita la cantidad de HO• en las ARM (Rodríguez-Chueca et al., 2014)but the solar UVA energy dose required to achieve similar results at pH 5 (24-30kJ/L, alcanzando un 38.5\% de degradación. Disminuyendo la eliminación del producto farmacéutico en comparación al FEFS, que alcanzó un 63.9\% de degradación (Tabla 1). De igual manera, 
el proceso FFS se pudo ver limitado por la eficiencia en la regeneración del $\mathrm{Fe}^{2+}$ (pH cercano a 8) (Babuponnusami \& Muthukumar, 2014). De otra parte, la corriente aplicada en el sistema FEFS, puede haber regenerado de nuevo el $\mathrm{Fe}^{2+}$ (M. D. G. De Luna et al., 2012; Ting et al., 2009)6-Dimethylaniline (2,6-DMA, lo que permitió mayor generación de radicales hidroxilo durante el tratamiento.

En términos de degradación, el proceso FCS removió 92.6\% de FLX (Gutierrez-Mata et al., 2017; Méndez-Arriaga et al., 2011). Un porcentaje mayor al FEFS y FFS, sin embargo, al momento de pensar en una aplicación real, se prefiere un proceso como FFS que no requiere separación del catalizador y funciona eficientemente a concentraciones de Fe por debajo de los límites permitidos por la norma. A su vez, implementar este proceso, obliga a buscar condiciones óptimas para mejorar la respuesta a pH natural.

\section{Conclusiones}

El proceso FFS demuestra mayor velocidad y eficiencia de degradación de la FLX cuando se encuentra en AD. En este proceso el pH ácido del medio, promueve mayor mineralización dado que a éste $\mathrm{pH}$ se mantiene una concentración elevada de radicales $\mathrm{OH}^{*}$ por encima de los que se promueven a pH natural. El proceso FCS resulta ser eficiente en la degradación y mineralización de la FLX, sin embargo, la cinética es lenta en comparación a los otros procesos. El proceso FEFS requiere el uso de un ánodo no activo como por ejemplo BDD, para promover la formación y fisisorción de radicales $\mathrm{OH}^{*}$ en su superficie capaces de ocasionar mineralización de FLX.

La cinética y la velocidad de degradación del proceso FFS, disminuye cuando FLX en degradada ARM. Este comportamiento se atribuye a la presencia de otros compuestos orgánicos e inorgánicos, que compiten por los radicales hidroxilos. Por otra parte, la degradación en el proceso FEFS no se ve disminuida, probablemente debido a que la corriente aplicada ocasiona la regeneración del $\mathrm{Fe}^{2+}$, per- mitiendo la producción de radicales hidroxilos. De los tres procesos el FCS, genera mayor porcentaje de degradación, sin embargo, la separación del catalizador del medio de reacción es una limitante al momento de pensar en una aplicación real.

Con la degradación de la FLX se generan subproductos que presentan menor toxicidad para organismos vivos como la levadura de cerveza (s. cerevisiae). La luz solar en todos los casos funciona en la fotoactivación de procesos catalíticos, permitiendo contemplar su uso en la optimización de procesos de degradación y mineralización de contaminantes emergentes presentes en aguas contaminadas reales.

\section{Agradecimientos}

A la Universidad de la Amazonia por la financiación del proyecto 600.6.519 - "Eliminación de un contaminante emergente presente en aguas residuales municipales de Florencia Caquetá, a partir de procesos fisicoquímicos avanzados" en el marco de la convocatoria interna de grupos del año 2016. El grupo GIRAB agradece a la Universidad de Antioquia UdeA y al CODI (UdeA) por el proyecto 201715947 - "Nanotubos modificados con FeOx para eliminación de actividad antimicrobiana".

\section{Referencias}

Affam, A. C., Chaudhuri, M., Kutty, S. R. M., \& Muda, K. (2014). UV Fenton and sequencing batch reactor treatment of chlorpyrifos, cypermethrin and chlorothalonil pesticide wastewater. International Biodeterioration \& Biodegradation, 93, 195-201. https://doi.org/10.1016/j.ibiod.2014.06.002

Agudelo, D. R. (2007). Degradación de colorantes de aguas residuales empleando UV/TiO2/H2O2/Fe2+. Universidad EAFIT, 42(146), 80-101.

Babuponnusami, A., \& Muthukumar, K. (2014). A review on Fenton and improvements to the Fenton process for wastewater treatment. Journal of Environmental Chemical Engineering, 2(1), 557-572. https://doi. org/10.1016/j.jece.2013.10.011

Bandala, V. E. E. R., Bautista, P., Mohedano, A. F., Casas, J. A., Zazo, J. A., Rodriguez, J. J., Brillas, E., Brooks, B. 
W., Foran, C. M., Richards, S. M., Weston, J., Turner, P. K., Stanley, J. K., Solomon, K. R., Slattery, M., La, T. W., Buxton, G. V, Greenstock, C. L., Helman, P., ... Wang, Y. (2010). Degradation of fifteen emerging contaminants at microg L(-1) initial concentrations by mild solar photo-Fenton in MWTP effluents. Water Research, 44(2), 545-554. https://doi.org/10.1016/j. watres.2009.09.059

Castro, A. De, Concheiro, M., Quintela, Ó., \& Cruz, A. (2005). Detección de fluoxetina y su metabolito activo norfluoxetina mediante LC-MS en plasma y saliva . Revista de Toxicología, 22(Su1), 142-149.

De la Cruz, N., Giménez, J., Esplugas, S., Grandjean, D., De Alencastro, L. F., \& Pulgarín, C. (2012). Degradation of 32 emergent contaminants by UV and neutral photo-fenton in domestic wastewater effluent previously treated by activated sludge. Water Research, 46(6), 1947-1957. https://doi.org/10.1016/j.watres.2012.01.014

De Luna, M. D. G., Veciana, M. L., Su, C. C., \& Lu, M. C. (2012). Acetaminophen degradation by electro-Fenton and photoelectro-Fenton using a double cathode electrochemical cell. Journal of Hazardous Materials, 217-218, 200-207. https://doi.org/10.1016/j. jhazmat.2012.03.018

Feng, H. E., \& Le-cheng, L. E. I. (2004). Degradation kinetics and mechanisms of phenol in photo-Fenton process * 5(2), 198-205.

Flores, N., Sirés, I., Garrido, J. A., Centellas, F., Rodríguez, R. M., Cabot, P. L., \& Brillas, E. (2015). Degradation of trans-ferulic acid in acidic aqueous medium by anodic oxidation, electro-Fenton and photoelectro-Fenton. Journal of Hazardous Materials, 1-10. https://doi.org/10.1016/j.jhazmat.2015.11.040

Giraldo-Aguirre, A. L., Erazo-Erazo, E. D., Flórez-Acosta, O. A., Serna-Galvis, E. A., \& Torres-Palma, R. A. (2015). TiO2 photocatalysis applied to the degradation and antimicrobial activity removal of oxacillin: Evaluation of matrix components, experimental parameters, degradation pathways and identification of organics by-products. Journal of Photochemistry and Photobiology A: Chemistry, 311, 95-103. https:// doi.org/10.1016/j.jphotochem.2015.06.021

Giraldo, A. L., Peñuela, G. a., Torres-Palma, R. a., Pino, N. J., Palominos, R. a., \& Mansilla, H. D. (2010). Degradation of the antibiotic oxolinic acid by photocatalysis with TiO2 in suspension. Water Research,
44(18), 5158-5167. https://doi.org/10.1016/j.watres.2010.05.011

Gutierrez-Mata, A. G., Velazquez-Martínez, S., ÁlvarezGallegos, A., Ahmadi, M., Hernández-Pérez, J. A., Ghanbari, F., \& Silva-Martínez, S. (2017). Recent Overview of Solar Photocatalysis and Solar PhotoFenton Processes for Wastewater Treatment. International Journal of Photoenergy, 1-27. https://doi. org/10.1155/2017/8528063

Honda, V., Silva, O., Paula, A., Silva, A. C., Teixeira, C., Borrely, S. I., Carlos, A., \& Costa, S. (2016). Degradation and acute toxicity removal of the antidepressant Fluoxetine ( Prozac ${ }^{\circledR}$ ) in aqueous systems by electron beam irradiation. Environmental Science and Pollution Research, 11927-11936. https://doi. org/10.1007/s11356-016-6410-1

Klamerth, N., Malato, S., Agüera, A., \& Fernández-Alba, A. (2013). Photo-Fenton and modified photo-Fenton at neutral $\mathrm{pH}$ for the treatment of emerging contaminants in wastewater treatment plant effluents: A comparison. Water Research, 47(2), 833-840. https://doi.org/10.1016/j.watres.2012.11.008

Li, W., Nanaboina, V., Zhou, Q., \& Korshin, G. V. (2011). Effects of Fenton treatment on the properties of effluent organic matter and their relationships with the degradation of pharmaceuticals and personal care products. Water Research, 46(2), 403-412. https:// doi.org/10.1016/j.watres.2011.11.002

Luna, R. A., Zermeño, B. B., Moctezuma, E., Contreras, R. E., Leyva, E., \& López B, M. A. (2013). FOTODEGRADACIÓN DE OMEPRAZOL EN SOLUCIÓN ACUOSA UTILIZANDO TiO2 COMO CATALIZADOR. Revista Mexicana de Ingeniería Química, 12(1), 85-95.

Méndez-Arriaga, F., Otsu, T., Oyama, T., Gimenez, J., Esplugas, S., Hidaka, H., \& Serpone, N. (2011). Photooxidation of the antidepressant drug Fluoxetine (Prozac () in aqueous media by hybrid catalytic/ozonation processes. Water Research, 45(9), 2782-2794. https:// doi.org/10.1016/j.watres.2011.02.030

Perini, J. A. D. L., Costa, B., Tonetti, A. L., \& Nogueira, R. F. P. (2016). Photo-Fenton degradation of the pharmaceuticals ciprofloxacin and fluoxetine after anaerobic pre-treatment of hospital effluent. Environmental Science and Pollution Research. https://doi. org/10.1007/s11356-016-7416-4

Rodríguez-Chueca, J., Polo-López, M. I., Mosteo, R., Ormad, M. P., \& Fernández-Ibáñez, P. (2014). Disinfection 
of real and simulated urban wastewater effluents using a mild solar photo-Fenton. Applied Catalysis B: Environmental, 150-151, 619-629. https://doi. org/10.1016/j.apcatb.2013.12.027

Salazar, C., Ridruejo, C., Brillas, E., Y????ez, J., Mansilla, H. D., \& Sir??s, I. (2017). Abatement of the fluorinated antidepressant fluoxetine (Prozac) and its reaction by-products by electrochemical advanced methods. Applied Catalysis B: Environmental, 203, 189-198. https://doi.org/10.1016/j.apcatb.2016.10.026

Salazar, C., Ridruejo, C., Brillas, E., Yáñez, J., Mansilla, H. D., \& Sirés, I. (2016). Abatement of the fluorinated antidepressant fluoxetine ( Prozac) and its reaction by electrochemical advanced methods. "Applied Catalysis B, Environmental." https://doi.org/10.1016/j. apcatb.2016.10.026

Serna-galvis, E. A., Silva-agredo, J., Giraldo, A. L., Flórezacosta, O. A., \& Torres-palma, R. A. (2016). Comparative study of the effect of pharmaceutical additives on the elimination of antibiotic activity during the treatment of oxacillin in water by the photoFenton, TiO 2 -photocatalysis and electrochemical processes. Science of the Total Environment, The, 541, 1431-1438. https://doi.org/10.1016/j.scitotenv.2015.10.029

Su, C., Bellotindos, L. M., Chang, A., \& Lu, M. (2013). Degradation of acetaminophen in an aerated Fenton reactor. Journal of the Taiwan Institute of Chemical Engineers, 44(2), 309-315. https://doi.org/10.1016/j. jtice.2012.11.009

Ting, W. P., Lu, M. C., \& Huang, Y. H. (2009). Kinetics of 2,6-dimethylaniline degradation by electro-Fenton process. Journal of Hazardous Materials, 161(23), 1484-1490. https://doi.org/10.1016/j.jhazmat.2008.04.119

Villegas-Guzman, P., Silva-Agredo, J., González-Gómez, D., Giraldo-Aguirre, A. L., Flórez-Acosta, O., \& TorresPalma, R. a. (2015). Evaluation of water matrix effects, experimental parameters, and the degradation pathway during the $\mathrm{TiO} 2$ photocatalytical treatment of the antibiotic dicloxacillin. Journal of Environmental Science and Health. Part A, Toxic/ Hazardous Substances \& Environmental Engineering, 50(1), 40-48. https://doi.org/10.1080/10934 529.2015 .964606

Zhao, Y., Yu, G., Chen, S., Zhang, S., Wang, B., Huang, J., \& Wang, Y. (2017). Ozonation of antidepressant fluoxetine and its metabolite product norfluoxitine : Kinetics , intermediates and toxicity. Chemical Engineering Journal. https://doi.org/10.1016/j. cej.2017.02.032
Manrique Losada, L.; Quimbaya Ñañez, C.; Torres Palma, R.A. (2019). Eliminación de fluoxetina presente en aguas contaminadas usando procesos fotoquímicos de oxidación avanzada y luz solar. Revista EIA, 16(32), Julio-Diciembre, pp. 27-40. [Online]. Disponible en: https://doi.org/10.24050/ reia.v16i32.1081 